\title{
NEAR COMPACTNESS AND SEPARABILITY OF SYMMETRIZABLE SPACES
}

\author{
R. M. STEPHENSON, JR.
}

\begin{abstract}
Although every feebly compact, Baire, semimetrizable space is separable, we prove here that for every infinite cardinal number $n$ there exists a feebly compact, Baire, symmetrizable Hausdorff space which has no dense subset of cardinality less than $n$.
\end{abstract}

For a topological space $X$, a mapping $d: X \times X \rightarrow[0, \infty)$ is said to be a symmetric provided that: (i) for all $x, y \in X, d(x, y)=d(y, x)$, and $d(x, y)$ $=0$ if and only if $x=y$; and (ii) for any subset $V$ of $X, V$ is open if and only if for each point $v \in V$ there exists $e>0$ with $B(v, e) \equiv\{x \in X: d(x, v)<$ $e\} \subset V$. If, in addition, each $B(x, e), x \in X, e>0$, is a neighborhood of $x$, then $d$ is called a semimetric. A space $X$ which has a symmetric (semimetric) is said to be symmetrizable (semimetrizable).

A. V. Arhangel'skii [A, p. 126] proved that every countably compact symmetrizable Hausdorff space is metrizable, and in [S1] and [S2] properties of symmetrizable feebly compact spaces were studied (recall that a space $X$ is said to be feebly compact if every locally finite family of open subsets of $X$ is finite). Of particular interest there was the question: Is every feebly compact symmetrizable space separable? Proofs were given in [S1] that every feebly compact symmetrizable space having a dense set of isolated points is separable, and in [S2, Theorem 10] that every feebly compact, Baire, semimetrizable space is separable. The latter extended Reed's theorem [R] that every Moore-closed space is separable, for a Moore-closed space is regular and feebly compact [G], and a regular, feebly compact space is Baire [M].

In this paper, a modification of a very nice technique developed in [DGN, Example 3.1] is used to settle the question in the negative, and we obtain the following surprising result.

THEOREM. Let $n$ be an infinite cardinal number. Then there exists a Baire, feebly compact, symmetrizable Hausdorff space $X$ such that no dense subset of $X$ has cardinality less than $m=n \aleph_{0}$.

Proof. Let $Y$ be a metrizable Baire space such that $|V|=m$ for every nonempty open subset $V$ of $Y$, and $|D|=m$ for any dense subset $D$ of $Y$. Let $d^{\prime}$ be a metric for $Y, \mathscr{B}$ a base for $Y$ with $|\mathscr{B}|=m$, and $\mathrm{C}$ be the family of all

Received by the editors June 20, 1977 and, in revised form, August 1, 1977.

AMS (MOS) subject classifications (1970). Primary 54E25, 54D55; Secondary 54D30.

Key words and phrases. Symmetrizable, feebly compact, Baire space. 
countably infinite, pairwise disjoint, locally finite families of nonempty members of $\mathscr{B}$.

List the members of the collection $\mathbf{C}$ as $\mathbf{C}=\left\{\bigodot_{k}: k<m\right\}$ and list the members of each $C_{k}$ in a 1-1 manner as $C_{k}=\left\{C_{k j}: j \in \mathbf{N}\right\}$. Since each $\left|C_{k, j}\right|=m$, one can by transfinite induction select points $s_{k, j} \in C_{k, j}$, where $k<m$ and $j \in \mathbf{N}$, so that whenever $i, k \in m$ and $i \neq k$, then

$$
\left\{s_{i, j}: j \in \mathbf{N}\right\} \cap\left\{s_{k, j}: j \in \mathbf{N}\right\}=\varnothing \text {. }
$$

Let $X=Y \cup m$ and extend $d^{\prime}$ to a symmetric $d$ on $X$ by the rule

$$
d(x, y)=d(y, x)= \begin{cases}0 & \text { if } x=y ; \\ d^{\prime}(x, y) & \text { if } x, y \in X \\ 1 / j & \text { if } x=k \text { and } y=s_{k, j} ; \text { and } \\ 1 & \text { otherwise. }\end{cases}
$$

Next, let $X$ have the topology induced on it by $d$.

Before verifying that $X$ is Hausdorff, observe that for each point $y \in Y$, one has $y=s_{k, j}$ for at most one pair $k, j$, so for each $y \in Y$ there exists $e(y)>0$ with $B(y, e(y)) \subset Y$. Thus $\{B(y, e): 0<e \leqslant e(y)\}$ is a fundamental system of open neighborhoods of $y$ in $X$. For a point $k<m$, a fundamental system of open neighborhoods is the family of all sets having the form

$$
\{k\} \cup\left(\cup\left\{B\left(s_{k, j}, f_{j}\right): 0<f_{j} \leqslant e\left(s_{k j}\right), t \leqslant j\right\}\right),
$$

where $t \in \mathbf{N}$ and $f$ is a sequence of real numbers, the $j$ th term of which is $f_{j}$.

Consider distinct points $x$ and $y$ in $X$. If both are in the metrizable open subset $Y$, then disjoint neighborhoods can certainly be found. Suppose $x=k<m$ and $y \in Y$. For some $t \in \mathbf{N},\{y\}$ and $\left\{s_{k j}: j \geqslant t\right\}$ are disjoint closed subsets of $Y$ (since $\mathcal{C}_{k}$ is locally finite in $Y$ and pairwise disjoint), so there exist disjoint open subsets $U$ and $V$ of $Y$ with $y \in U$ and $\left\{s_{k j}\right.$ : $j \geqslant t\} \subset V$. Thus, $U$ and $V \cup\{k\}$ are disjoint neighborhoods of $y$ and $x$. If $x=k$ and $y=r$ with $k, r<m$, then one can (again) appeal to the normality of $Y$ and topology on $X$ to find disjoint open sets containing $\{k\} \cup\left\{s_{k, j}\right.$ : $j \in \mathbf{N}\}$ and $\{r\} \cup\left\{s_{r, j}: j \in \mathbf{N}\right\}$.

Because $Y$ a dense, Baire subspace of $X$, the space $X$ must also be Baire. Since $Y$ is an open subspace having no dense subset of cardinality less than $m$, then $X$ has no dense subset of cardinality less than $m$.

Finally, suppose that $\mathfrak{V}$ is an infinite family of open subsets of $X$. We will prove that $\mathcal{V}$ fails to be locally finite.

Suppose, on the contrary, that $\mathscr{V}$ is locally finite. Since $Y$ is dense in $X$, one can find a countably infinite pairwise disjoint family $\mathcal{W}$ of members of $\mathscr{B}$ and a 1-1 mapping $f: \mathcal{W} \rightarrow \mathcal{T}$ such that for each $W \in \mathscr{W}$, one has $W \subset$ $f(W)$. Evidently any point at which $\mathcal{W}$ fails to be locally finite must also be a point at which $\mathcal{V}$ fails to be locally finite. Thus $\mathscr{W}$ is locally finite with respect to $Y$, and hence $\mathscr{W}=\mathcal{C}_{k}$ for some $k<m$. But clearly $\bigodot_{k}$ fails to be 
locally finite at the point $k$, so we have a contradiction.

REMARKS. (i) I do not know if every regular, feebly compact symmetrizable space is separable. Since a $G_{\delta}$-point in a regular, feebly compact space must have a countable neighborhood base (by an observation of I. Glicksberg), and since a first countable symmetrizable Hausdorff space is semimetrizable, any example of a regular, feebly compact, symmetrizable space that is not separable would also provide a negative answer to the still open question (see [DGN]) as to whether or not every point of a regular symmetrizable space must be a $G_{\delta}$.

(ii) In the construction above, if $Y$ is chosen so that no compact subset of $Y$ has nonempty interior, then arguments similar to ones given in [DGN] show that $X$ has a closed subset, namely $m$, which fails to be a $G_{\delta}$-set (because then if $\mathcal{V}$ is a countable family of open sets containing $m$, the family $\mathscr{W}=\{V \cap$ $Y: V \in \mathcal{V}\}$ consists of dense open subsets of $Y$, and so $\varnothing \neq \cap \otimes \subset Y$ and $\cap \mathcal{V} \neq m)$.

\section{BIBLIOGRAPHY}

[A] A. V. Arhangel'skii, Mappings and spaces, Usephi Mat. Nauk 21 (1966), no. 4 (130), 133-184 = Russian Math. Surveys 21 (1966), 115-162. MR 37 \#3534.

[DGN] S. W. Davis, G. Gruenhage and P. J. Nyikos, $G_{\delta}$-sets in symmetrizable and related spaces, General Topology and Appl. (to appear).

[G] J. W. Green, Moore-closed spaces, completeness and centered bases, General Topology and Appl. 4 (1974), 297-313. MR 51 \# 1754.

[M] R. A. McCoy, A filter characterization of regular Baire spaces, Proc. Amer. Math. Soc. 40 (1973), 268-270. MR 49 \#3859.

[R] G. M. Reed, On chain conditions in Moore spaces, General Topology and Appl. 4 (1974), 255-267. MR 49 \#11481.

[S1] R. M. Stephenson, Jr., Symmetrizable, $\mathscr{F}_{-}$, and weakly first countable spaces, Canad. J. Math. 29 (1977).

[S2] Symmetrizable-closed spaces, Pacific J. Math. 70 (1977).

Department of Mathematics and Computer Science, University of South Carolina, Columbia, SOUTh Carolina 29208 